\title{
Electrical Resistivity of CrN Thin Films
}

\begin{abstract}
I. BATKO ${ }^{a, *}$, M. BATKOVA ${ }^{a}$, F. LOFAJ ${ }^{b}$
${ }^{a}$ Institute of Experimental Physics, Slovak Academy of Sciences, Watsonova 47, 04001 Košice, Slovakia

${ }^{b}$ Institute of Materials Research, Slovak Academy of Sciences, Watsonova 47, 04001 Košice, Slovakia

The work is focused on the measurements of electrical resistivity of $\mathrm{CrN}$ thin films deposited on glass substrates by DC-magnetron sputtering in $\mathrm{Ar}+\mathrm{N}_{2}$ atmosphere. The studied samples reveal semiconducting behaviour of electrical resistivity in the whole range of tested preparation parameters (such as pressure and composition of Ar$\mathrm{N}_{2}$ mixture), whereas the electrical transport regime is strongly influenced by parameters of preparation. Numerical analysis of the experimental data showed that electrical transport can be adequately described in terms of variablerange hopping conduction in selected temperature intervals. Moreover, S-shaped anomaly in $\rho(T)$ dependence, being expected to be a consequence of phase transition to a low-temperature antiferromagnetic orthorhombic phase, has been observed for sample with the highest concentration of $\mathrm{N}_{2}$ in the temperature interval of $220-250 \mathrm{~K}$. The obtained results indicate that technology processes typically used for preparation of CrN coatings represent a promising potential to develop also high sensitivity cryogenic sensors for high magnetic fields applications.
\end{abstract}

DOI: $10.12693 /$ APhysPolA.126.415

PACS: 72.80.Ga, 72.20.Ee, 78.30.Hv

\section{Introduction}

Chromium nitride $(\mathrm{CrN})$ has received a considerable attention due to its high mechanical hardness, hightemperature stability and corrosion resistance that are important for applications in hard, wear, and corrosion resistant coatings [1]. In addition, $\mathrm{CrN}$ attracts increasing interest as a potential electronic and spintronic material due to its magnetic ordering and possible band gap, which makes it a promising material for diluted magnetic semiconductors, particularly when combined with (or doped into) other nitride semiconductors $[2,3]$. Another interesting application of $\mathrm{CrN}$ lies in the field of thermometry, since $\mathrm{CrN}$ thin films fabricated by RFmagnetron sputtering were reported as prospective temperature sensors for cryogenic region with small magnetoresistance [4].

Electrical properties of $\mathrm{CrN}$ represent a controversial point as they show metallic behaviour with $d \rho / d T>0$ in some studies, as well as a qualitatively opposite behaviour, $d \rho / d T<0$, in another reports [5]. Moreover, $\rho(T)$ curves of $\mathrm{CrN}$ frequently show a discontinuity at 260-280 K [6], which is associated with a magnetic and structural phase transition from a paramagnetic $\mathrm{NaCl}$ structure to a low-temperature antiferromagnetic orthorombic $P_{m n a}$ phase at Néel temperature $T_{N}=273$ $286 \mathrm{~K}$ [2]. However, epitaxial $\mathrm{CrN}(001)$ layers, grown by $\mathrm{DC}$-magnetron sputtering on $\mathrm{MgO}(001)$ substrates at growth temperatures $T_{s}$ of $550-850{ }^{\circ} \mathrm{C}$, did not show any discontinuity in $\rho(T)$ at $\sim 280 \mathrm{~K}$, which suggests that epitaxial constraints suppress the phase transition to the low-temperature antiferromagnetic orthorhombic phase [2]. Moreover, these epitaxial $\mathrm{CrN}(001)$ layers exhibit electronic transport dominated by variable-range

*corresponding author; e-mail: batko@saske.sk hopping (VRH) with a transition from Efros-Shklowskii type to Mott type VRH at $30 \pm 10 \mathrm{~K}$ [2].

The aim of this work is to investigate electrical properties of $\mathrm{CrN}$ thin films prepared by reactive magnetron sputtering as a function of deposition conditions to check their application potential as cryogenic thermometers.

\section{Experimental details}

CrN thin films (coatings) with the thickness of 380$410 \mathrm{~nm}$ were deposited from 3" Cr target using reactive magnetron sputtering with the balanced DC-magnetron with the power of $500 \mathrm{~W}$ on a PVD apparatus (model Cryofox Discovery 500, Polyteknik, Denmark). The reactive sputtering was done in $\mathrm{Ar}+\mathrm{N}_{2}$ atmosphere under total pressure of 0.5 and $1 \mathrm{~Pa}$ with the nitrogen content in the interval of 30-50\% of total gas flow. All the films were deposited on glass substrates at ambient temperature. Measurements of electrical resistivity and magnetoresistance were performed by means of four-probe method in a commercial cryo-magnetic system (Physical Property Measurement System (PPMS), Quantum Design, USA).

\section{Results and discussion}

Temperature dependences of the resistivity for three selected $\mathrm{CrN}$ thin films are shown in Fig. 1. (Parameters of the deposition process are indicated in the figure.) All the studied films reveal semiconducting behaviour of electrical resistivity. $\rho(T)$ dependence of the $\mathrm{CrN}$ film grown at $30 \%$ of nitrogen reveals a pronounced S-shaped anomaly at temperature of 220-250 K, expected to be a consequence of phase transition to the low-temperature antiferromagnetic orthorhombic phase [2]. A slight sign for S-shaped anomaly can be found also at the resistivity curve of the film grown at $40 \%$ of $\mathrm{N}_{2}$. The phase transition to the low-temperature phase is probably completely suppressed in films reactively sputtered at sufficiently high concentration of $\mathrm{N}_{2}$, because the sample deposited at the highest proven concentration of nitrogen (50\%) does not show any visible anomaly of the resistivity in the whole investigated temperature range. Such 
observation is in good agreement with the work reported on $\mathrm{CrN}$ thin films fabricated by $\mathrm{RF}$ magnetron sputtering in pure $\mathrm{N}_{2}$ atmosphere that also did not reveal the anomaly [4].

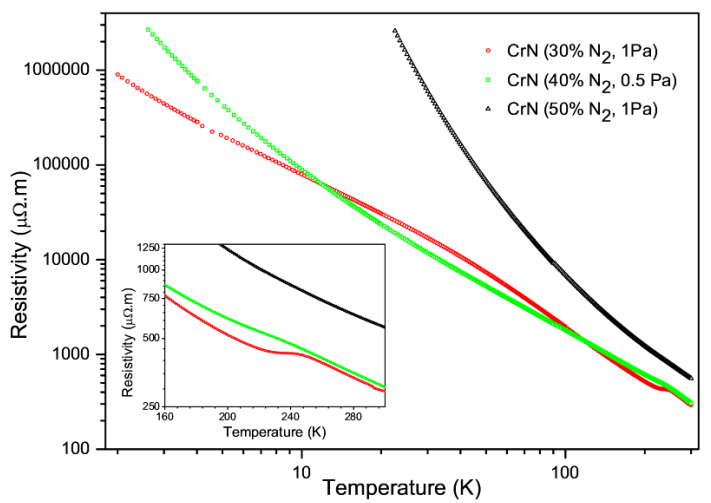

Fig. 1. $\rho(T)$ dependences of $\mathrm{CrN}$ thin films deposited in $\mathrm{Ar}+\mathrm{N}_{2}$ atmosphere with $\mathrm{N}_{2}$ concentration of $30 \%$ (blue circles), $40 \%$ (red squares), and $50 \%$ (black triangles).

The analysis of the experimental data indicate that electrical transport can be adequately described within the Efros-Shklowskii scenario of VRH $\left(\rho=\rho_{0} \exp \left[\left(T_{0} / T\right)^{1 / 2}\right]\right) \quad[7]$ at the lowest temperatures, while intervals obeying Mott's law of VRH $\left(\rho=\rho_{0} \exp \left[\left(T_{0} / T\right)^{1 / 4}\right]\right)[7,8]$ can be found above $260 \mathrm{~K}$ for all investigated samples. Such results are in good qualitative agreement with the investigations of other authors [2], who also reported crossover between Mott and Efros-Shklowskii type of VRH.

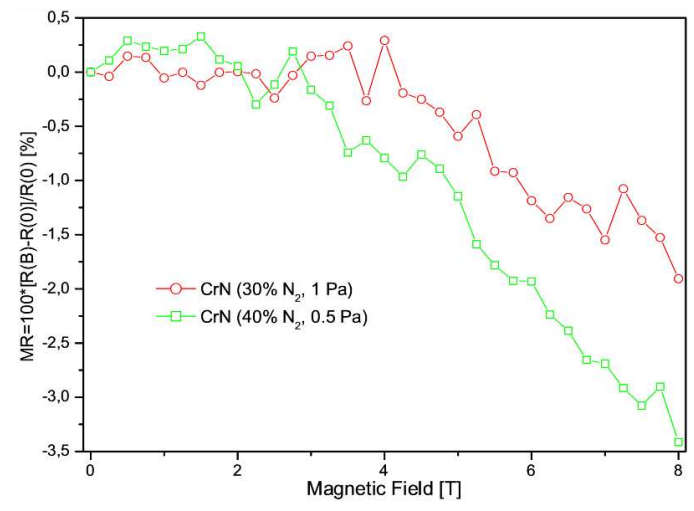

Fig. 2. Magnetoresistance of selected $\mathrm{CrN}$ thin films measured at temperature of $4 \mathrm{~K}$.

Figure 2 shows that the magnetoresistance of $\mathrm{CrN}$ thin films prepared in atmosphere with $30 \%$ and $40 \% \mathrm{~N}_{2}$ is lower than $3.5 \%$ and $1.9 \%$ at $4 \mathrm{~K}$ and magnetic field up to $8 \mathrm{~T}$, respectively. Considering to use such thin $\mathrm{CrN}$ films for thermometry purposes in magnetic fields, the mentioned resistivity changes would correspond to an error of temperature determination less than $1.7 \%$ (30\% of $\mathrm{N}_{2}$ ) or $0.7 \%\left(40 \%\right.$ of $\mathrm{N}_{2}$ ) it the mentioned temperature and magnetic field range. Taking into account that the increase of $\mathrm{N}_{2}$ concentration increases the sensitivity and decreases the magnetoresistance, the obtained results indicate a possibility of the development of $\mathrm{CrN}$-based temperature sensors with very low temperature shift due to magnetoresistance.

\section{Conclusions}

Based on the obtained results it can be concluded that electrical properties of $\mathrm{CrN}$ thin films prepared by $\mathrm{DC}$ magnetron sputtering can be effectively controlled by the deposition conditions such as total gas pressure and composition of $\mathrm{Ar}+\mathrm{N}_{2}$ mixture. An S-shaped anomaly in $\rho(T)$ dependence was observed at temperature of 220$250 \mathrm{~K}$ for the sample prepared with the least concentration of $\mathrm{N}_{2}$. Numerical analysis of the experimental data indicates that electrical transport of all studied samples can be adequately described in terms of variablerange hopping conduction in selected temperature intervals. Relatively small magnetoresistance of the $\mathrm{CrN}$ thin films prepared by sputtering in atmosphere with lower $\mathrm{N}_{2}$ concentration suggests an effective technology approach to control properties of $\mathrm{CrN}$ thin films for their use as temperature sensors for cryogenic region and high magnetic fields.

\section{Acknowledgments}

This work was supported by the Slovak Scientific Agency VEGA (contract Nos. 2-0184-13 and 2/0108/11), by the Slovak Research and Development Agency (contract Nos. APVV-0520-10 and APVV-0034-07), and by the European Union European Regional Development Fund (contr. Nos. ITMS 26220120005 and ITMS $26220120035)$.

\section{References}

[1] B. Navišek, P. Panjan, I. Milošev, Surf. Coat. Technol. 97, 182 (1997).

[2] X.Y. Zhang, J.S. Chawla, B.M. Howe, D. Gall, Phys. Rev. B 83, 165205 (2011).

[3] A. Ney, J. Rajaram, S.S.P. Parkin, T. Kammermeier, S. Dhar, Phys. Rev. B 76, 035205 (2007).

[4] T. Yotsuya, Y. Takehi, T. Ishida, Cryogenics 51, 546 (2011).

[5] X.Y. Zhang, D. Gall, Phys. Rev. B 82, 045116 (2010).

[6] K. Inumaru, K. Koyama, N. Imo-Oka, S. Yamanaka, Phys. Rev. B 75, 054416 (2007).

[7] A.L. Efros, B.I. Shklovskii, Electronic Properties of Doped Semiconductors, 2nd ed., Clarendon, Oxford, 1979.

[8] N.F. Mott, E.A. Davis, Electronic Precesses in NonCrystalline Materials, 2nd ed., Clarendon, Oxford, 1979. 